\title{
A strategy for Precision Measurements of the W-boson Mass and Width at the LHC
}

\author{
K. Rejzner* \\ II. Institut für Theoretische Physik, Universität Hamburg, Hamburg, Germany. \\ Marian Smoluchowski Institute of Physics, Jagiellonian University, Krakow, Poland. \\ E-mail: katarzyna.rejzner@mail.desy.de \\ F. Fayette \\ LPNHE, Pierre et Marie Curie Universités Paris VI et Paris VII, Paris, France. \\ I. Physikalisches Institut, Universität Göttingen, Göttingen, Germany.
}

\section{W. Krasny}

LPNHE, Pierre et Marie Curie Universités Paris VI et Paris VII, Paris, France.

\section{W. Płaczek}

Marian Smoluchowski Institute of Physics, Jagiellonian University, Krakow, Poland.

\section{A. Siódmok}

Marian Smoluchowski Institute of Physics, Jagiellonian University, Krakow, Poland. LPNHE, Pierre et Marie Curie Universités Paris VI et Paris VII, Paris, France. Institut für Theoretische Physik, Universität Karlsruhe, Germany.

\begin{abstract}
The strategies to measure the electroweak model parameters at the Tevatron and at LHC must be different. There are two basic reasons for that: (1) the Tevatron is the proton-antiproton collider while the LHC is the proton-proton one, (2) LHC collider energy is large enough to open the "Pandora box" of heavy quark-flavour degrees of freedom, which are poorly controlled. We shall demonstrate, in this presentation, that the present precision of the SM parameters cannot be improved by the LHC programme, unless LHC-dedicated measurement methods are used and a "precision-support" experimental program is conducted. We shall focus our attention on the measurement of the mass of the $W$-boson, its charge asymmetry and on the measurement of the $W$-boson width. We shall propose a dedicated measurement program, specific to the LHC environment, capable to improve the present precision of these parameters.
\end{abstract}

XXth Hadron Collider Physics Symposium

November 16 - 20, 2009

Evian, France

${ }^{*}$ Speaker. 


\section{Measurement simulation}

The tool for event generation is WINHAC 1.30 - a Monte Carlo generator for single- $W$ production in hadronic collisions with its leptonic decays. WINHAC includes also neutral-current processes, with $\gamma$ and $Z$ bosons in the intermediate state, at the Born level. A distinct feature of the WINHAC event generator is that it describes $W$ and $Z$ production and decay in terms of spin amplitudes. The advantage of this approach is that one has explicit control over all spin states, and thus over transverse and longitudinal boson polarization and their interferences.

The events have been simulated using a parametrized average response of the detector. The systematic biases in the detector response have been modeled and simulated in the dedicated generation and simulation runs.

More detailed discussions of these measurement strategies are presented in Refs. [1, 2, 3, 4, 5].

\section{Observables}

We assume in the following that the mass of the $W$-boson will be determined using the following observables:

$$
\begin{aligned}
& O_{W^{+}}\left(p_{T, l}, \eta_{l}\right)=\frac{d^{2} \sigma}{d p_{T, l^{+}} d \eta_{l^{+}}}\left(“ l^{+} \text {events }^{\prime \prime}\right), \quad O_{W^{-}}\left(p_{T, l}, \eta_{l}\right)=\frac{d^{2} \sigma}{d p_{T, l}-d \eta_{l^{-}}}\left(“ l^{-} \text {events" }\right), \\
& O_{Z}\left(M_{l l}, y_{l l}, p_{T, l l}\right)=\frac{d^{3} \sigma}{d M_{l l} d y_{l l} d p_{T, l}}\left(“ l^{+} l^{-} \text {events" }\right), \\
& O_{Z^{+}}\left(M_{l l}, p_{T, l}, \eta_{l}\right)=\frac{d^{3} \sigma}{d M_{l l} d p_{T, l^{+}} d \eta_{l^{+}}}\left(“ l^{+} l^{-} \text {ev." }\right), \quad O_{Z^{-}}\left(M_{l l}, p_{T, l}, \eta_{l}\right)=\frac{d^{3} \sigma}{d M_{l l} d p_{T, l^{-}} d \eta_{l^{-}}}\left(“ l^{+} l^{-} \text {ev." }\right),
\end{aligned}
$$

where $p_{T, l^{+}}, \eta_{l^{+}}\left(p_{T, l^{-}}, \eta_{l^{-}}\right)$denote, respectively, the transverse momentum and pseudorapidity of the positively (negatively) charged lepton, $M_{l l}, y_{l l}$ and $p_{T, l l}$ are, respecively, the mass, rapidity and transverse momentum of the lepton pair. The signature of the " $l^{+}$events" (" $l{ }^{-}$events") is the presence of precisely one isolated $l^{+}\left(l^{-}\right)$candidate. The signature of the " $l^{+} l^{-}$ev." is the presence of the two isolated opposite charge lepton candidates.

We assume that the observables are uncorrected for the calibration of the positive and of the negative lepton momentum scale, specified in terms of the calibration factors: $\varepsilon_{l^{+}}$and $\varepsilon_{l^{-}}$. These two parameters describe the relationship between the true and the reconstructed curvature radius of positive and negative tracks in the solenoid magnetic field of the LHC detectors: $\rho_{l^{+(-)}}^{\text {rec. }}=\rho_{l^{+(-)}}^{\text {true }} \times$ $\left(1+\varepsilon_{l^{+(-)}}\right)$. In order to measure the $W$-boson mass, the observables defined in (2.1) must be interpreted within the Standard Model:

$$
\begin{aligned}
& O_{W^{+}}\left(p_{T, l}, \eta_{l}\right)=T_{W^{+}}\left(\varepsilon_{l^{+}}, u, d, s, c, \bar{u}, \bar{d}, \bar{s}, \bar{c}, M_{W^{+}}, \Gamma_{W^{+}}\right) \\
& O_{W^{-}}\left(p_{T, l}, \eta_{l}\right)=T_{W^{-}}\left(\varepsilon_{l^{-}}, u, d, s, c, \bar{u}, \bar{d}, \bar{s}, \bar{c}, M_{W^{-}}, \Gamma_{W^{-}}\right) \\
& O_{Z}\left(M_{l l}, y_{l l}, p_{T, l l}\right)=T_{Z}\left(\varepsilon_{l^{-}}, \varepsilon_{l^{+}}, u, d, s, c, b, \bar{u}, \bar{d}, \bar{s}, \bar{c}, \bar{b}, M_{Z}, \Gamma_{Z}, \sin ^{2} \theta_{W}, \alpha\right) \\
& O_{Z^{+}}\left(M_{l l}, p_{T, l}, \eta_{l}\right)=T_{Z^{+}}\left(\varepsilon_{l^{+}}, u, d, s, c, b, \bar{u}, \bar{d}, \bar{s}, \bar{c}, \bar{b}, M_{Z}, \Gamma_{Z}, \sin ^{2} \theta_{W}, \alpha\right) \\
& O_{Z^{-}}\left(M_{l l}, p_{T, l}, \eta_{l}\right)=T_{Z^{-}}\left(\varepsilon_{l^{+}}, u, d, s, c, b, \bar{u}, \bar{d}, \bar{s}, \bar{c}, \bar{b}, M_{Z}, \Gamma_{Z}, \sin ^{2} \theta_{W}, \alpha\right)
\end{aligned}
$$

where, $u, d, s, c, b, \bar{u}, \bar{d}, \bar{s}, \bar{c}, \bar{b}$ are the $k_{T}$-unintegrated PDFs, $M_{W^{+}}, M_{W^{-}}, M_{Z}$ are the masses of the $W$ and of the $Z$ bosons, $\Gamma_{W^{+}}, \Gamma_{W^{-}}, \Gamma_{Z}$ are the respective widths, $\theta_{W}$ is the Weinberg angle and $\alpha$ is the fine-structure constant. The functional forms of $T_{X}$ are those implemented in the WINHAC/ZINHAC generators. 
We propose to base the measurements of the Standard Model parameters on the following experimental observables:

$$
\begin{array}{llll}
\operatorname{Asym}_{W}^{(+,-)}\left(p_{T, l}, \eta_{l}\right) & =\frac{O_{W^{+}}\left(p_{T, l}, \eta_{l}\right)-O_{W^{-}}\left(p_{T, l}, \eta_{l}\right)}{O_{W^{+}}\left(p_{T, l}, \eta_{l}\right)+O_{W^{-}}\left(p_{T, l}, \eta_{l}\right)}, & \operatorname{Asym}_{Z}^{(+,-)}\left(p_{T, l}, \eta_{l}\right)=\frac{O_{Z^{+}}\left(p_{T, l}, \eta_{l}\right)-O_{Z^{-}}\left(p_{T, l}, \eta_{l}\right)}{O_{Z^{+}}\left(p_{T, l}, \eta_{l}\right)+O_{Z^{-}}\left(p_{T, l}, \eta_{l}\right)}, \\
\operatorname{R}_{W Z}\left(p_{T, l}, \eta_{l}\right) & =\frac{O_{W^{+}}\left(p_{T, l}, \eta_{l}\right)+O_{W^{-}}\left(p_{T, l}, \eta_{l}\right)}{O_{Z^{+}}\left(p_{T, l}, \eta_{l}\right)+O_{Z^{-}}\left(p_{T, l}, \eta_{l}\right)}, & \mathrm{R}_{Z}^{\text {norm }}\left(p_{T, l}, \eta_{l}\right) & =\frac{O_{Z^{+}}\left(p_{T, l}, \eta_{l}\right)+O_{Z^{-}}\left(p_{T, l}, \eta_{l}\right)}{O_{l^{+}}^{\text {norm }}},
\end{array}
$$

where

$$
\begin{array}{lll}
O_{Z^{+(-)}}\left(p_{T, l}, \eta_{l}\right) & = & \int_{M_{Z}-3 \Gamma_{Z}}^{M_{Z}+3 \Gamma_{Z}} O_{Z^{+(-)}}\left(M_{l l}, p_{T, l}, \eta_{l}\right) d M_{l l}, \\
O_{l^{+} l^{-}}^{\text {norm }} & = & \iiint O_{Z}\left(M_{l l}, y_{l l}, p_{T, l l}\right) d M_{l l} d y_{l l} d p_{T, l l} .
\end{array}
$$

The last integral is over the phase-space of the coplanar $l^{+} l^{-}$pairs produced in peripheral $p p$ collisions.

\section{Unfolding biases}

We used the following method of unfolding biases:

- First we generated a pseudo-data event sample $\mathscr{P} \mathscr{D}$, representing a given systematic bias $\xi$ (for a fixed value of the physical parameter $\mathscr{P}$ ref, where $\mathscr{P}=M_{W}, \Gamma_{W}, \ldots$ ).

- Then we generated a set of $2 k+1$ unbiased (i.e. $\xi=0$ ) template data samples $\mathscr{M} \mathscr{T}$. Each sample $n$ of the $\mathscr{M} \mathscr{T}$ set corresponds to a given value of $\mathscr{P}^{(n)}=\mathscr{P}^{\mathrm{ref}}+\delta \mathscr{P}^{(n)}$

- The likelihood between the binned $d \sigma / d p_{T, l}$ (or $d \sigma / d \eta_{l}$ ) distributions for the $n^{\text {th }} \mathscr{M} \mathscr{T}$ sample and the bias-dependent $\mathscr{P} \mathscr{D}$ sample was quantified in terms of the $\chi^{2}$ value:

$$
\chi^{2}\left(p_{T, l} ; \xi, n\right)=\sum_{i} \frac{\left(d \sigma_{i ; \xi}-d \sigma_{i ; \xi=0, n}\right)^{2}}{\Delta d \sigma_{i ; \xi}^{2}+\Delta d \sigma_{i ; \xi=0, n}^{2}} .
$$

\begin{tabular}{|l|c|c|c|}
\hline & $\begin{array}{c}\Delta\left[\Gamma_{W}\right] \\
\mathrm{MeV}\end{array}$ & $\begin{array}{c}\Delta\left[\left(M_{W^{+}}+M_{W^{-}}\right) / 2\right] \\
\mathrm{MeV}\end{array}$ & $\begin{array}{c}\left.\Delta\left(M_{W^{+}}-M_{W^{-}}\right)\right] \\
\mathrm{MeV}\end{array}$ \\
\hline$c^{\text {bias }}=0.9 \cdot c, \quad s^{\text {bias }}=s+0.1 \cdot c$ & +32 & +148 & +17 \\
\hline$c^{\text {bias }}=1.1 \cdot c, \quad s^{\text {bias }}=s-0.1 \cdot c$ & -12 & -111 & -11 \\
\hline \hline$u_{v}^{\text {bias }}=0.95 \cdot u_{v}, d_{v}^{\text {bias }}=d_{v}+0.05 \cdot u_{v}$ & -10 & -64 & -139 \\
\hline$u_{v}^{\text {bias }}=1.05 \cdot u_{v}, d_{v}^{\text {bias }}=d_{v}-0.05 \cdot u_{v}$ & +10 & +79 & +115 \\
\hline \hline$b^{\text {bias }}=1.2 \cdot b$ & +43 & +42 & 0 \\
\hline$b^{\text {bias }}=0.8 \cdot b$ & -34 & -39 & 0 \\
\hline
\end{tabular}

Table 1: The results of the performed analysis for some chosen biases.

Our analysis has shown that the main source of errors for the precision measurements are uncertainties of PDF's. We have used a simple model to take those uncertainties into account. We have assumed the coherent shifts between quarks of different flavors. For example, we have checked the influence of decreasing the number of $c$ quarks by $10 \%$ with the simultaneous increasing of the 
number of $s$ quarks (see Table 1). It has been shown that effects caused by heavy flavour quarks are very significant and without applying some new strategy the present precision of measurement of $W$ mass and width cannot be improved at the LHC.

\section{The way forward}

The precision of the $M_{W}$ and $\Gamma_{W}$ can be improved if one applies a dedicated strategy which would take into account all the effects characteristic for a $p p$ collider. Such a strategy was proposed by our group and it has following main steps:

- The LHC runs should be performed not only for the $p p$ collisions but also $d d$ collisions. Use of isoscalar beams has many advantages. Among them:

- Isoscalar beams $u_{v}=d_{v}$ cancel the majority of $W^{+}, W^{-}$and $Z$ production differences,

- The measurement of the $W$-boson charge-asymmetry constraint can be achieved directly from the $s-c$ distribution.

- There should be dedicated runs with rescaled collision CM energy: $\sqrt{s_{1}}$ and $\sqrt{s_{2}}=\frac{M_{Z}}{M_{W}} \cdot \sqrt{s_{1}}$. These two settings allow to keep the momentum fractions of the partons producing the $Z$ and $W$-bosons equal, if the $W$-boson sample is collected at the CM-energy $\sqrt{s_{1}}$ and the $Z$-boson sample at the CM-energy $\sqrt{s_{2}}$.

- There should be dedicated runs with reversed solenoid magnetic field. The solenoid current should be rescaled while running at the two CM-energies $\sqrt{s_{1}}$ and $\sqrt{s_{2}}$ by a factor $\frac{i_{2}}{i_{1}}=\frac{M_{Z}}{M_{W}}$ to equalize the distribution of the curvature radius $\rho_{l}$ for charged leptons originating from the decays of the $Z$ and $W$ bosons.

- An "LHC-precision-support" programme has been proposed: a DIS experiment with COMPASS. Use of $W$ and $Z$ data only is not sufficient for constraining the PDF's. External constraints are needed. An optimal (in terms of its complementarities to the LHC measurements) constraint can be provided by a COMPASS high-precision measurement. The Letter of Intent for such an experiment has been submitted to the SPSC and LHCC [6].

\section{References}

[1] M. W. Krasny, F. Fayette, W. Placzek and A. Siodmok, 'Z-boson as 'the standard candle' for high precision W-boson physics at LHC”, Eur. Phys. J. C 51 (2007) 607; arXiv: hep-ph/0702251.

[2] F. Fayette, M. W. Krasny, W. Placzek and A. Siodmok, "Measurement of $M W^{+}-M W^{-}$at $L H C$ ", Eur. Phys. J. C 63 (2009) 33; arXiv: 0812.2571 [hep-ph].

[3] F. Fayette, "Strategies for precision measurements of the charge asymmetry of the W boson mass at the LHC within the ATLAS experiment", arXiv: 0906.4260 [hep-ex].

[4] A. Siodmok, F. Fayette, M. W. Krasny, W. Placzek "W-boson mass measurement: from the Tevatron to the $L H C$ ", in preparation.

[5] M.W. Krasny, J. Chwastowski and K. Slowikowski, Nucl. Instrum. Meth. A584 (2008) 42.

[6] F. Dydak, M.W. Krasny and R. Voss, CERN-SPSC-2009-028/SPSC-I-239, CERN-LHCC-2009-014/LHCC-I-017, September 2009. 